\title{
Controversies in the management of gastroschisis: a study of 40 patients
}

\author{
M D Stringer, R J Brereton, V M Wright
}

\begin{abstract}
Forty infants with gastroschisis were referred to two paediatric surgeons during a 13 year period. Overall survival was $90 \%$. Nine patients were transferred in utero and 31 were referred postnatally. Birth weights, gestational ages, and Apgar scores were similar for both groups. Primary closure of the defect was successfully achieved in seven $(\mathbf{7 8 \%})$ patients in the prenatally transferred group compared with $17(55 \%)$ in the postnatal group. Significantly less postoperative assisted ventilation, and a trend in favour of early discharge home, were noted after prenatal transfer. Problems arising during postnatal transfer may have contributed to these differences. No major differences resulting from the mode of delivery were identified. Patients treated by primary closure fared significantly better than those undergoing staged repairs with prosthetic material. Prospective randomised studies are required to confirm these findings.
\end{abstract}

The incidence of gastroschisis is approximately 1/10 000 births. In both Europe and the United States there has been an apparent increase in incidence during the past decade. ${ }^{1}$ Major controversies affecting the obstetrician, neonatologist, and paediatric surgeon surround the management of gastroschisis. Uncertainty clouds the relative merits of prenatal compared with postnatal transfer, caesarean section compared with vaginal delivery, and primary compared with serial closure techniques. This retrospective study of $\mathbf{4 0}$ recently treated neonates attempts to define significant differences in outcome between these groups of patients.
Hospitals for Sic Children and University College Hospital,

$M$ D Stringer

J Brereton

Correspondence to: Mr M D Stringer, Department of Paediatric Surgery, Institute of Child Health, 30 Guilford Street, London WCIN $1 \mathrm{EH}$

Accepted 21 August 1990

\section{Patients and methods}

A retrospective analysis was undertaken of the case notes of a consecutive series of 40 neonates with gastroschisis treated during the past 13 years. In some cases this was supplemented by the obstetrician's notes and interviews with mothers. Those transferred prenatally were delivered and underwent surgery at a single institution whereas those transferred postnatally underwent surgery at either of the two Hospitals for Sick Children or at University College Hospital, London. Primary abdominal wall closure was performed whenever possible but a staged repair with a prosthetic patch or silo was employed when necessary.

The median maternal age was 21 years (range 17-31) and 29 mothers were primiparous, which accords with known epidemiological data. ${ }^{1}$ Nine infants underwent surgery after prenatal transfer (group 1) and 31 after postnatal referral (group 2).

Statistical analyses were by the $\chi^{2}$ test, the unpaired $t$ test for normally distributed data, or the Mann-Whitney $U$ test for non-parametric data.

\section{Results}

Mean gestational ages, birth weights, and Apgar scores at 5 minutes were similar in both groups (table 1). Successful primary closure was achieved in $7 / 9(78 \%)$ patients in group 1 compared with $17 / 31(55 \%)$ in group 2 (no significant difference using the $\chi^{2}$ test with Yates's correction). Patients in group 1 tended to undergo surgery sooner after birth, were ventilated for significantly less time, and tended to be discharged home earlier than those in group 2 (table 1).

Although only nine (23\%) patients were referred prenatally, gastroschisis had been detected prenatally in $21(53 \%)$. Successful primary closure was achieved in $12(57 \%)$ infants diagnosed prenatally and in nine (47\%) of those who had not been detected prenatally. There was no significant difference in the frequency of primary closure after delivery by caesarean section $(8 / 11)$ compared with that after vaginal delivery (16/29) and there were no significant differences in the three measures of postoperative morbidity. Four of the 11 caesarean sections were performed electively because of the prenatal diagnosis of gastroschisis, two were for breech presentation, and the remainder were for fetal distress. There was no association between the extent of evisceration (small bowel and colon with or without stomach

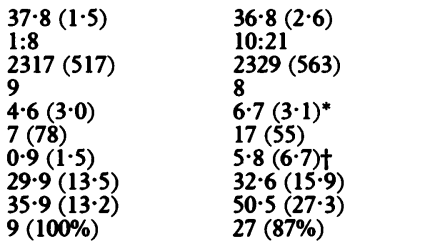


Table 2 Comparison of primary and serial closure groups

\begin{tabular}{|c|c|c|c|}
\hline & $\begin{array}{l}\text { Primary } \\
\text { closure } \\
(n=21)\end{array}$ & $\begin{array}{l}\text { Serial } \\
\text { closure } \\
(n=12)\end{array}$ & Significance \\
\hline $\begin{array}{l}\text { Mean (SD) gestational age (weeks) } \\
\text { Mode of delivery (caesarean:vaginal) } \\
\text { Mean birth weight (g) } \\
\text { Median Apgar scores (5 min) } \\
\text { Mean (SD) age at operation (hours) } \\
\text { No (SD) days of postoperative ventilation } \\
\text { No (SD) days to full enteral feeding } \\
\text { No (SD) days total parenteral nutrition } \\
\text { No (SD) days hospital stay }\end{array}$ & $\begin{array}{l}36 \cdot 6(2 \cdot 8) \\
7: 14 \\
2296(625) \\
9 \\
6 \cdot 2(3 \cdot 8) \\
3 \cdot 9(6 \cdot 1) \\
26 \cdot 7(12 \cdot 9) \\
21 \cdot 1(13 \cdot 2) \\
37 \cdot 3(17 \cdot 6)\end{array}$ & $\begin{array}{l}37 \cdot 7(1 \cdot 6) \\
3: 9 \\
2350(410) \\
9 \\
6 \cdot 1(2 \cdot 2) \\
7 \cdot 2(12 \cdot 3) \\
40 \cdot 9(15 \cdot 0) \\
32 \cdot 4(14 \cdot 4) \\
62 \cdot 8(28 \cdot 2)\end{array}$ & $\begin{array}{l}\text { NS } \\
\text { NS } \\
\text { NS } \\
\text { NS } \\
\text { NS } \\
\text { NS } \\
\text { p }<0.02 \\
\text { p }<0.05 \\
\text { p }<0.02\end{array}$ \\
\hline
\end{tabular}

$\mathrm{n}=33$; excludes four postoperative deaths and three survivors with associated intestinal atresia.

and pelvic viscera) and the ability of the surgeon to effect successful primary closure.

Excluding the four postoperative deaths and the three survivors with associated intestinal atresia, patients undergoing primary closure were ventilated for no longer than those receiving staged repair but achieved full enteral feeding significantly earlier and were discharged home sooner (table 2 ).

Associated congenital anomalies were uncommon (table 3) being detected in only 13 (33\%). No chromosomal abnormalities were identified in the four tested patients with multiple anomalies.

Several neonates undergoing postnatal transfer arrived in poor condition (table 4). Bilious vomiting, due to an absent or inadequately sited/sized nasogastric tube, occurred in six, but fortunately none of these had evidence of pulmonary aspiration. The cooling of 'warm' saline packs used to cover the eviscerated intestine resulted in appreciable hypothermia in five and uncorrected fluid and plasma losses from the exposed intestine demanded urgent colloid resuscitation in four neonates.

Table 3 Associated congenital anomalies in 13 patients with gastroschisis*

Gastrointestinal:

Jejunoileal atresias (two high jejunal atresias, one ileal,
and one apple peel variant) and one apple peel

Other anomalies:

Undescended testes (two unilateral and two bilateral) 4

$\begin{array}{ll}\text { Undescended testes (two unilateral and two bilateral) } & 4 \\ \text { Inguinal hernias (one unilateral and one bilateral) } & 2\end{array}$

Arthrogryposis multiplex with congenital hip dislocation 1

*Errors of intestinal rotation have been excluded.

Some infants had more than one associated anomaly.

Table 4 Complications of management in 40 infants with gastroschisis

\begin{tabular}{ll}
\hline Complications of management & $\begin{array}{l}\text { No of } \\
\text { infantst }\end{array}$ \\
\hline $\begin{array}{l}\text { Complications during postnatal transfer: } \\
\text { Inadequate nasogastric drainage }\end{array}$ & 6 \\
Hypothermia (core temperature $\leqslant 35 \cdot 0^{\circ} \mathrm{C}$ ) & 5 \\
Appreciable hypovolaemia & 4 \\
Major postoperative complications: & \\
Bowel ischaemia/necrosis after primary closure & 2 \\
Partial dehiscence or sepsis of patch/silo & 5 \\
Cannula/long line sepsis & 5 \\
Necrotising enterocolitis & 4 \\
Convulsions & 4 \\
Long term complications* & 7 \\
Failure to thrive (<3rd centile at 1 year) & 4 \\
Incisional hernias & 4 \\
Symptomatic gastro-oesophageal reflux & 3 \\
\hline
\end{tabular}

${ }^{*}$ Eight infants under the age of 1 year and four early deaths are excluded.

tSome infants had more than one complication.
The overall initial survival rate after gastroschisis repair was $90 \%$. Of the four deaths, two were due in part to bowel ischaemia after 'tight' primary closure. Subsequent attempts to redeem the situation with a silo were unsuccessful. One death was from status epilepticus in an infant who had required massive intestinal resection and stomas for gastroschisis complicated by midgut volvulus necessitating prolonged intravenous nutrition complicated by hypocalcaemia, septicaemia, and disseminated intravascular coagulation. The remaining patient died aged 6 weeks from extensive neonatal necrotising enterocolitis after staged silo repair. There was one late death from acute bronchiolitis in a 6 week old infant who had been discharged home three weeks after an uneventful primary repair.

\section{Discussion}

There are three outstanding controversies in the management of neonates with gastroschisis: firstly, whether prenatal transfer confers any advantage to the prenatally diagnosed patient; secondly, whether the patient should be delivered vaginally or by caesarean section; and thirdly, whether the anterior abdominal wall defect is optimally managed by primary or serial closure. It is difficult to resolve these questions by a retrospective study but until prospective randomised trials have been undertaken, retrospective analyses provide the only source of data for clinical guidelines.

Despite statistical limitations, there was a clear trend in the prenatal transfer group in favour of more frequent successful primary closure, less postoperative ventilation, and reduced hospital stay. There are many possible reasons for this, including earlier surgery, but a likely major factor is the preoperative condition of the infant, as this is an important determinant of postoperative outcome. ${ }^{2}$ Hypothermia, hypovolaemia, and inadequate nasogastric drainage were readily identifiable complications of postnatal transfer leading to delays in surgery. Several layers of 'cling-film' wrapped around the infant successfully preserved central temperature and reduced evaporative losses whereas the use of saline soaked swabs resulted in rapid cooling. Transparent plastic sheeting also allows ready inspection of the bowel and the early recognition of intestinal ischaemia, which was noted on arrival in two of our patients. It is uncertain as to whether or not the postnatal transfer of infants under optimal conditions would eliminate the apparent advantages of prenatal transfer. A protocol designed to minimise the hazards of postnatal transfer of the infant with gastroschisis is suggested in table 5. The maternal morbidity resulting from 'in utero transfer' should be recognised, but could not be analysed in this study. ${ }^{34}$

The obstetric management of gastroschisis has been discussed by several authors. ${ }^{5-7}$ In the present study, there were no discernable differences in outcome between infants delivered by caesarean section and those delivered vaginally and it is accepted that there is greater maternal morbidity associated with delivery by caesarean section. ${ }^{8}$ Until prospective randomised trials 
Table 5 A protocol for postmatal transfer in gastroschisis

-Early liaison with neonatal surgical unit
-Adequately sited size 8-10 French gauge nasogastric tube on free
drainage with aspiration every 15 minutes
- Double 'cling-film' wrap of the abdomen including exposed
intestine
-Colloid infusion ( $10-20 \mathrm{ml} / \mathrm{kg}$ )
- Broad spectrum prophylactic antibiotics
- Meticulous temperature control in transport incubator
- Consent for surgery/vitamin $\mathrm{K}$ intramuscularly/maternal blood
sample
- Rapid transport of the infant after initial resuscitation

have been conducted, we would concur with others that vaginal delivery does not appear to adversely affect outcome, ${ }^{7-11}$ and that the mode and timing of delivery should be determined by indications other than the presence of a fetal abdominal wall defect.

The surgical management of the abdominal wall defect has generated much discussion among paediatric surgeons. Attitudes range from those who advocate primary closure whenever possible because of the higher mortality and morbidity rates of staged repair (principally sepsis, sac separation, and the need for multiple operative procedures) ${ }^{12-16}$ to those who recommend serial closure with prosthetic material because of the hazards of tight primary closure (principally intestinal ischaemia, respiratory and renal impairment). ${ }^{17-20}$ In this study primary closure was attempted whenever it was considered to be safely possible. Adopting this policy based on subjective clinical judgment, infants undergoing primary closure were more quickly established on full enteral feeding and discharged home significantly earlier than those treated by staged repair. ${ }^{21} 22$

Two of the four deaths were partly related to excessively tight primary closure resulting in intestinal ischaemia but nevertheless, intestinal infarction may occur as a complication of staged repair. ${ }^{18}$ Large defects with gross visceroabdominal disproportion clearly require staged repair. ${ }^{23}$ Intraoperative assessment of intraabdominal pressure, pulmonary compliance, or haemodynamic parameters may provide valuable objective information that would optimise closure technique in borderline cases. $^{24} 25$

This study confirms the generally good prognosis of infants with gastroschisis, 2101422 which is in sharp contrast to that of infants with exomphalos. ${ }^{1026}$ Major cardiac anomalies and abnormal karyotypes are unusual in infants with gastroschisis and associated abnormalities are principally gastrointestinal and amenable to corrective surgery. ${ }^{1026}$ Nevertheless, terminations of affected pregnancies are not uncommon. ${ }^{1026}$ Now that it is possible to distinguish between gastroschisis and exomphalos using prenatal ultrasonography in major antenatal diagnostic centres, ${ }^{9}$ guidelines for the termination of pregnancy need to be reconsidered. The retrospective data in this study suggest that when feasible primary closure may be advantageous, that there appears to be no benefit from delivery by caesarean section and that prenatal transfer may be associated with improved results. When prenatal transfer is impractical, optimum postnatal transfer is mandatory (table 5). Prospective randomised trials are the only method of satisfactorily addressing these issues.

We wish to acknowledge the skill and dedication of our anaesthetic and nursing colleagues involved in the care of these patients.

1 Torfs C, Curry C, Roeper P. Gastroschisis. I Pediatr 1990;116:1-6.

2 Muraji T, Tsugawa C, Nishijima E, Tanano H, Matsumoto Y, Kimura K. Gastroschisis: a 17-year experience. $\mathcal{f}$ Pediatr Surg 1989;24:343-5.

3 Ryan TDR, Kidd GM. Maternal morbidity associated with in utero transfer. BMF 1989;299:1383-5.

4 Crowley P, Lamont R, Elder MG. The obstetric care of the fetus transferred in utero. Foumal of Obstetrics and Gymecology 1982;2:129-33.

5 Lenke RR, Hatch EI. Fetal gastroschisis: a preliminary report advocating the use of cesarian section. Obstet Gynecol 1986;67:395-8.

6 Kirk EP, Wah RM. Obstetric management of the fetus with omphalocele or gastroschisis: a review and report of one hundred twelve cases. Am $\mathcal{F}$ Obstet Gymecol 1983;146: 512-6.

7 Carpenter MW, Curci MR, Dibbins AW, Haddow JE. Perinatal management of ventral wall defects. Obstet Gynecol 1984;64:646-51.

8 Cunningham FG, MacDonald PC, Grant NF. Cesarean section and cesarian hysterectomy. Williams' obstetrics. 18th Ed. London: Prentice-Hall International, 1989:443-4.

9 Bond SJ, Harrison MR, Filly RA, Callen PW, Anderson RA, Golbus MS. Severity of intestinal damage in gastroschisis: correlation with prenatal sonographic findings. $\mathcal{J}$ Pediatr Surg 1988;23:520-5.

10 Lafferty PM, Emmerson AJ, Fleming PJ, Frank JD, Noblett HR. Anterior abdominal wall defects. Arch Dis Child 1989;64:1029-31.

11 Bethel CAI, Seashore JH, Touloukian RJ. Cesarian section does not improve outcome in gastroschisis. $\mathcal{F}$ Pediatr Surg 1989;24:1-4.

12 Stringel G, Filler RM. Prognostic factors in omphalocele and gastroschisis. F Pediatr Surg 1979;14:515-9.

13 Filston MC. Gastroschisis-primary fascial closure: the goal for optimal management. Ann Surg 1983;197:260-4.

14 Canty TG, Collins DL. Primary fascial closure in infants with gastroschisis and omphalocele: a superior approach. f Pediatr Surg 1983;18:707-12.

15 Di Lorenzo M, Yazbeck S, Ducharme JC. Gastroschisis: 15-year experience. $\mathcal{f}$ Pediatr Surg 1987;22:710-2.

16 Ein SH, Rubin SZ. Gastroschisis: primary closure or silon pouch. $\mathcal{f}$ Pediatr Surg 1980;15:549-52.

17 Allen RG, Wrenn EL. Silon as a sac in the treatment of omphalocele and gastroschisis. F Pediatr Surg 1969;4:3-8.

18 King DR, Savrin RA, Boles ET. Gastroschisis update. F Pediatr Surg 1980;15:553-7.

19 Stone HH. Immediate permanent fascial prosthesis for gastroschisis and massive omphalocele. Surg Gynecol Obstet gastroschisis and
$1981 ; 153: 221-4$.

20 Schwartz MZ, Tyson KRT, Milliorn K, Lobe TE. Staged reduction using a silastic sac is the treatment of choice for large congenital abdominal wall defects. $\mathcal{f}$ Pediatr Surg 1983;18:713-9.

21 Luck SR, Sherman JO, Raffensperger JG, et al. Gastroshisis in 106 consecutive newborn infants. Surgery 1985;98: 677-83.

22 Caniano DA, Brokaw B, Ginn-Pease ME. An individualized approach to the management of gastroschisis. $\mathcal{F}$ Pediat Surg 1990;25:297-300.

23 Fonkalsrud EW. Selective repair of neonatal gastroschisis based on degree of visceroabdominal disproportion. Ann Surg 1980;191:139-44.

24 Yaster M, Buck JR, Dudgeon DL, et al. Hemodynamic effects of primary closure of omphalocele/gastroschisis in human newborns. Anesthesiology 1988;69:84-8.

25 Yaster M, Scherer TLR, Stone M, et al. Prediction of successful primary closure of congenital abdominal wall defects using intraoperative measurements. $\mathcal{f}$ Pediatr Surg 1989;12:1217-20.

26 Mann L, Ferguson-Smith MA, Desai M, Gibson AAM, Raine PAM. Prenatal assessment of anterior abdominal wall defects and their prognosis. Prenat Diagn 1984;4: 427-35. 